\title{
Interleukin-22 attenuates allergic airway inflammation in ovalbumin-induced asthma mouse model
}

Jingru Wang ${ }^{1,2}$, Shengnan Gao ${ }^{2,3}$, Jingyuan Zhang ${ }^{2,3}$, Chunxiao Li ${ }^{1,2}$, Hongwen Li ${ }^{1,2}$ and Jiangtao Lin ${ }^{1,2^{*}}$

\begin{abstract}
Background: Allergic asthma is a chronic airway inflammatory disease with a number of cytokines participating in its pathogenesis and progress. Interleukin (IL)-22, which is derived from lymphocytes, acts on epithelial cells and play a role in the chronic airway inflammation. However, the actual role of IL-22 in allergic asthma is still unclear. Therefore, we explored the effect of IL-22 on allergic airway inflammation and airway hyperresponsiveness (AHR) in an ovalbumin (OVA)-induced asthma mouse model.

Methods: To evaluate the effect of IL-22 in an allergic asthma model, BALB/C mice were sensitized and challenged with OVA; then the recombinant mouse IL-22 was administered intranasally $24 \mathrm{~h}$ prior to each challenge. The IL-22 levels in lung homogenates and bronchoalveolar lavage fluid (BALF) were measured by enzyme linked immunosorbent assay, respectively. AHR was evaluated through indicators including airways resistance (Rrs), elastance (Ers) and compliance (Crs); the inflammatory cell infiltration was assessed by quantification of differential cells counts in BALF and lung tissues stained by hematoxylin and eosin (H\&E); IL-22 specific receptors were determined by immunohistochemistry staining.
\end{abstract}

Results: The concentration of IL-22 was significantly elevated in the OVA-induced mice compared with the control mice in lung homogenates and BALF. In the OVA-induced mouse model, IL-22 administration could significantly attenuate AHR, including Rrs, Ers and Crs, decrease the proportion of eosinophils in BALF and reduce inflammatory cell infiltration around bronchi and their concomitant vessels, compared with the OVA-induced group. In addition, the expression of IL-22RA1 and IL-10RB in the lung tissues of OVA-induced mice was significantly increased compared with the control mice, while it was dramatically decreased after the treatment with IL-22, but not completely attenuated in the IL-22-treated mice when compared with the control mice.

Conclusion: Interleukin-22 could play a protective role in an OVA-induced asthma model, by suppressing the inflammatory cell infiltration around bronchi and their concomitant vessels and airway hyperresponsiveness, which might associate with the expression of its heterodimer receptors. Thus, IL-22 administration might be an effective strategy to attenuate allergic airway inflammation.

Keywords: Allergic asthma, Cytokines, Interleukin-22, Animal model, Ovalbumin sensitization

*Correspondence: jiangtao_|@263.net

1 Peking University China-Japan Friendship School of Clinical Medicine,

No. 2, East Yinghua Road, Chaoyang Disteict, Beijing 100029, China

Full list of author information is available at the end of the article

\section{Background}

Asthma is one of the most common chronic airway inflammatory disorders that affects 358.2 million individuals worldwide, up by $12.6 \%$ [1]. The China Pulmonary Health $(\mathrm{CPH})$ study has reported that the 
overall prevalence of asthma was $4.2 \%$ in adults [2]. Allergic asthma is the most common subtype of asthma [3], characterized by chronic airway inflammation and hyperresponsiveness, affecting approximately $60 \%$ of all asthma patients [4]. Airway epithelial cells and immune cells are occupied in bidirectional communication to regulate the chronic airway inflammation [5]. Cytokines are involved in asthmatic chronic airway inflammation, especially those derived from the lymphocyte, which acts on epithelial cells, such as cytokine interleukin (IL)-22.

Interleukin-22 is a member of the IL-10 family, and predominately derives from innate and adaptive immune cells, including $\alpha \beta \mathrm{T}$ cells, $\gamma \delta \mathrm{T}$ cells, natural killer T cells and group 3 innate lymphoid cells (ILC3s) [6-9]. It functions as a bridge by binding to its type 2-cytokine receptor, which is composed of a heterodimer of IL-22 receptor A1 (IL-22RA1) and IL-10RB; the former is expressed on non-hematopoietic cells, especially epithelial cells, but not immune cells $[10,11]$, while the latter is expressed on hematopoietic and non-hematopoietic cells [11]. By combining with IL-22RA1 and IL-10RB, IL-22 activates target cells to participate in inflammation, antimicrobial immunity and tissue repair [12], possibly playing an important role in the chronic airway inflammation of asthma. IL22-binding protein (IL-22BP), also known as IL-22RA2, is a soluble form of the IL-22 receptor homolog, which binds IL-22 with high affinity and neutralizes the activity of IL-22 in vitro $[13,14]$.

IL-22 plays bidirectional roles in allergic airway inflammation, both protective and pathogenic. On the one hand, the concentration of IL-22 significantly increases in peripheral blood mononuclear cells (PBMC), serum and lung tissues of asthma patients compared with healthy controls [15-18]. In mouse models of asthma, the IL-22 level increases in the lung after airway challenge [19]. IL-22 could induce antimicrobial protein, Reg3y, produced by signal transducer and activator of transcription (STAT) 3 activation, which then reduced the secretion of IL-33 and thymic stromal lymphopoietin (TSLP) of the lung epithelial cells, and inhibited the eosinophilic airway inflammation [20]; IL-22 could also suppress proinflammatory cytokine interferon- $\gamma($ IFN- $-\gamma)$-induced secretion of proinflammatory chemokines in human bronchial epithelial cells in vitro [21], demonstrating that it may have a potential protective role in asthma. Besides, in IL-22-deficient (IL-22 ${ }^{-/}$) mice or administration anti-IL-22 antibody wild-type (WT) mice, allergic airway inflammation and airway hyperresponsiveness (AHR) was aggravated, manifested as an enhanced proportion of eosinophils in bronchoalveolar lavage fluid (BALF), exacerbated inflammatory cell infiltration around the bronchi and their concomitant vessels, increased airway responsiveness and cytokine production $[9,19,20]$, indicating the protective role of IL-22 in allergic airway inflammation. On the other hand, IL-22 deficiency or administration of antiIL-22 antibody could dramatically decrease the proinflammatory cytokine production, eosinophil recruitment, AHR and mucus secretion [22-24], suggesting IL-22 may play a pathogenic role in allergic airway inflammation. Therefore, the effects of IL-22 on allergic asthma airway inflammation still need further study.

In view of the nonalignment of IL-22 roles in allergic asthma airway inflammation, we set out to investigate the role of IL-22 in the pathogenesis of allergic airway inflammation in asthma mouse model.

\section{Methods}

Mice

Wild type female BALB/c mice (aged 6-8 weeks) were purchased from Vital River Laboratory (License No. SCXK (Jing) 2019-0009, Beijing, China). All BALB/c mice were randomly divided into different groups (10 mice per group), and were maintained in standard animal housing conditions (12 h light and $12 \mathrm{~h}$ dark cycles, and freely obtaining food and water) of Animal Laboratory of China-Japan Friendship Hospital. All protocols on mice were approved by the Animal Ethical and Welfare Committee of China-Japan Friendship Hospital (approval No. zryhyy21-20-01-3).

\section{Ovalbumin (OVA)-induced allergic airway inflammation}

The OVA-induced mouse model of asthma was established as described previously $[25,26]$. BALB/c mice were divided into three groups: the phosphate buffered saline (PBS) control group (control), ovalbumin group (OVA/PBS), and OVA + recombinant IL-22 group (OVA/ IL-22). BALB/c mice were anesthetized with isofluorane inhalation before the intranasal administration. At Days 0,7 , and 14, mice were sensitized by intraperitoneal injection with $100 \mu \mathrm{g}$ OVA (Sigma-Aldrich, St. Louis, MO, USA; grade V) and $2.25 \mathrm{mg}$ aluminum hydroxide in PBS or equivalent PBS (200 $\mu$ l per mouse); then they were challenged by inhaled OVA (100 $\mu \mathrm{g}$ in $50 \mu \mathrm{l}$ PBS per mouse) or equivalent PBS on Days 22, 24, and 26. The recombinant mouse IL-22 (rmIL-22, BD Biosciences, San Diego, Calif, USA) (0.2 $\mu$ g per mouse) (as the OVA/IL-22 group) or PBS (as the control and OVA/PBS group) was administered intranasally $24 \mathrm{~h}$ before the inhaled OVA challenge. As described previously with minor modifications [19, 22, 24, 27], we have chosen the dose of rmIL$22(0.2 \mu \mathrm{g}$ per mouse). The BALB/c mice were sacrificed $24 \mathrm{~h}$ after the last OVA challenge (Fig. 1a).

\section{Assessment of airway responsiveness}

Twenty-four hours after the last intranasal OVA challenge, airway responsiveness was assessed on day 27 


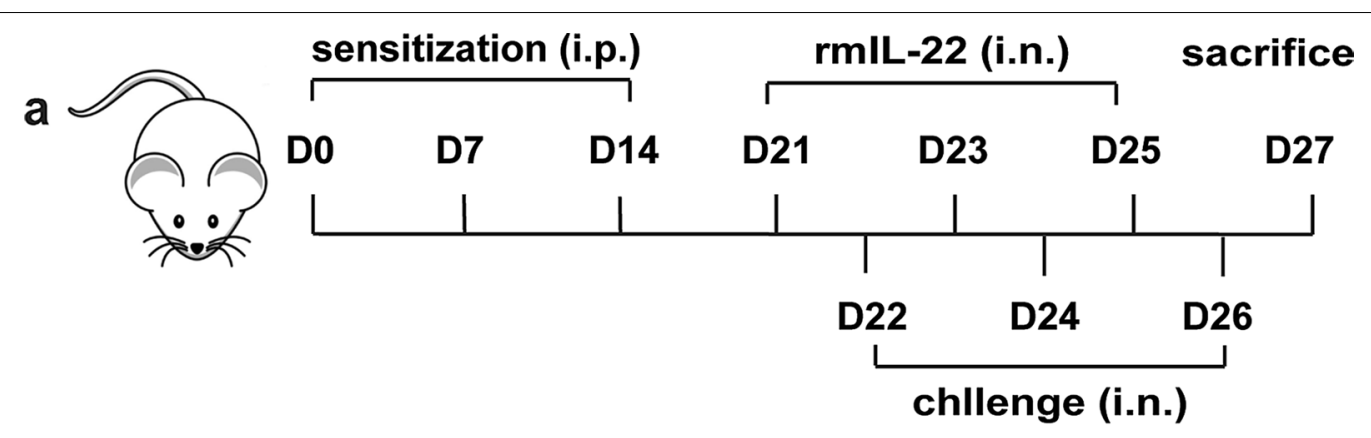

b

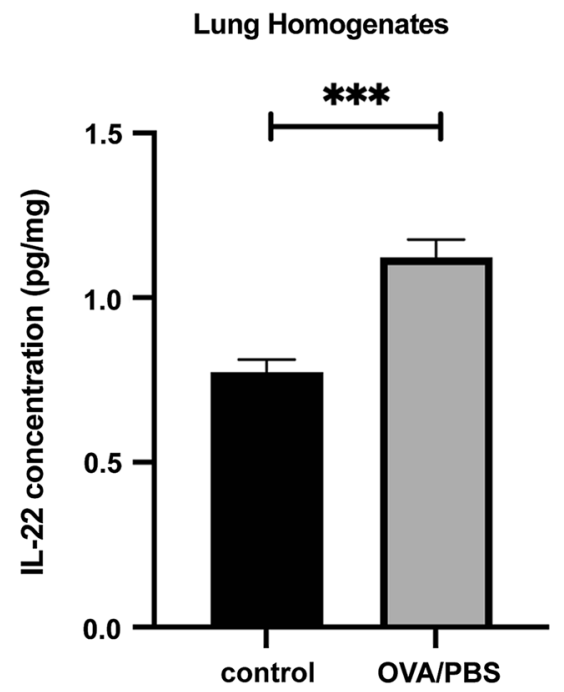

C

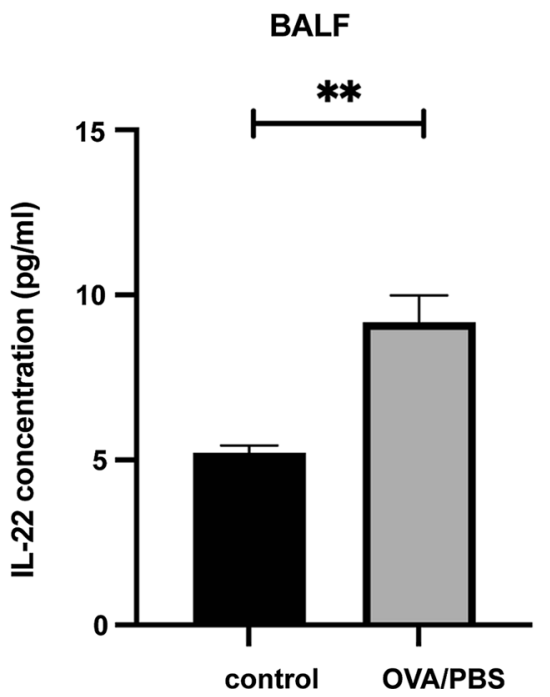

Fig. 1 The schedule for the OVA-induced asthma model and the expression of IL-22 in OVA-induced asthma model. a Schedule for the OVA-induced asthma model; $\mathbf{b}$ concentration of IL-22 in lung homogenates $(n=8)$; $\mathbf{c}$ concentration of IL-22 in BALF( $n=4-8)$. Bars are presented as the mean \pm SEM. i.n. intranasal, i.p. intraperitoneal; ${ }^{*} p<0.05,{ }^{* *} p<0.01,{ }^{* * *} p<0.001$

using the FlexiVent system (SCIREQ, Inc., Montreal, Canada) following the manufacturer's protocol, as previously described [28]. Briefly, after the anesthetization with $80 \mathrm{mg} / \mathrm{kg}$ pentobarbital sodium (Sigma-Aldrich, St. Louis, MO, USA) by intraperitoneal injection, mice were conducted with tracheotomy and endotracheal intubation. And then airway responsiveness was assessed by measuring the changes of lung resistance after sequentially exposed to increasing doses of methacholine (Mch, Sigma-Aldrich, St. Louis, MO, USA) in sterile saline (0, $6.25,12.5,25$ and $50 \mathrm{mg} / \mathrm{ml})$. The values of airways resistance (Rrs), elastance (Ers) and compliance (Crs) were recorded for analyzing the AHR.

\section{Collection of BALF and quantification of differential cells counts}

After the measurement of airway responsiveness, BALF was collected by instilling and retrieving $500 \mu \mathrm{l}$ of cold sterile saline into the lung tissues, and this above process was repeated three times [29]. And then BALF was centrifuged at $1500 \mathrm{~g}$ for $10 \mathrm{~min}$ at $4{ }^{\circ} \mathrm{C}$, supernatants were stored at $-80{ }^{\circ} \mathrm{C}$ for further use, whereas the cell pellets were resuspended in $50 \mu \mathrm{l}$ of sterile PBS to calculate differential cell counts (eosinophils, neutrophils, lymphocytes and macrophages) by Wright-Giemsa (BaSO, Zhuhai City, Guangdong, China) staining. And 400 cells were counted in each section in continuous fields of vision.

\section{Lung histopathology}

Following the collection of BALF, the mice were euthanized with pentobarbital sodium. Left lung lobes were fixed by $10 \%$ neutral-buffered formalin for $24 \mathrm{~h}$, and then embedded in paraffin. Finally, paraffin-embedded sections $(5 \mu \mathrm{m})$ were stained with haematoxylin/ eosin (H\&E) to assess the scores of inflammatory cells infiltration, as previously described [30]. The numbers of peribronchiolar and perivascular infiltrating 
inflammatory cells were scored as follows: 0 , no cells; 1 , a few cells; 2 , a ring of inflammatory cells, one cell layer of peribronchial cells; 3 , a ring of inflammatory cells, two to four cells layers of peribronchial cells; and 4, a ring of inflammatory cells, more than four cell layers of peribronchial cells. Independent operators ignorant of the origin of the sections performed. Five to ten airways were counted in per mouse.

\section{Lung immunohistochemistry}

Immunohistochemistry was used to determine the location of IL-22 specific receptor. After heated in incubator at $60{ }^{\circ} \mathrm{C}$ for $2 \mathrm{~h}$, paraffin-embedded sections were treated by immersion in xylene and gradient alcohol solutions for deparaffinization and hydration, respectively, and then by sinking in $10 \mathrm{mM}$ citrate buffer for antigen recovery. Then, after the endogenous peroxidase activity was blocked by $3 \% \mathrm{H}_{2} \mathrm{O}_{2}$, sections were administrated with $10 \%$ goat serum blocking solution to reduce the nonspecific absorption of immunoglobulin. Finally, the specimens were incubated with polyclonal antibodies, anti-IL-22RA1 antibody (1:500, Merk-Millipore, Temecula, CA, USA), anti-IL-10RB antibody (1:500, Bioss, Beijing, China), and anti-IL22BP antibody (1:500, Abcam, Cambridge, CB2 0AX, UK) at $4{ }^{\circ} \mathrm{C}$ for overnight, and then stained with a goat anti-rabbit IgG secondary antibody-HRP (1:500, Thermo Fisher Scientific, Rockford, IL, USA) at $37{ }^{\circ} \mathrm{C}$ for $30 \mathrm{~min}$, according to manufacturer's protocols. Image J software was used to evaluate protein expression. Ten digital photographs of bronchioles in each tissue section were observed under $40 \times$ magnification. Data were presented as a percentage of the positively stained area in whole segments of lung tissues.

\section{IL-22 expression in lung homogenates and BALF}

The right lung tissues were weighed and homogenized in cold PBS containing 2\% protease inhibitor and phosphatase inhibitors cocktail (Solarbio, Beijing, China) by highly speed homogenizer (IKA, Guangzhou, Guangdong, China). The lung homogenates were centrifugated at $4{ }^{\circ} \mathrm{C}, 14,000 \mathrm{~g}$ for $10 \mathrm{~min}$, then the sediments were removed and the supernatants were collected. The IL-22 levels in lung homogenates and BALF were determined by enzyme linked immunosorbent assay (ELISA) Kit (MULTI SCIENCES, Hangzhou City, Zhejiang, China). The procedures were executed following the manufacturer's instructions. The absorbance was measured at $450 \mathrm{~nm}$ and $570 / 630 \mathrm{~nm}$ by microplate spectrophotometer (Thermo Fisher Scientific, Waltham, MA USA) and data were expressed as $\mathrm{pg} / \mathrm{ml}$ (sensitivity $0.74 \mathrm{pg} / \mathrm{ml}$ ).

\section{Statistical analysis}

The data analyses and graphs preparation were performed by SPSS 20.0 (IBM, Armonk, New York, USA) and GraphPad Prism 9.0 (GraphPad, San Diego, CA, USA). When the sample size was less than 50, Kolmogorov-Smirnov was used to analyzed whether the experimental data obey the normal distribution. For normal distribution data, independent Student $t$ test or one-way analysis of variance (ANOVA) was used for comparing different groups; Wilcoxon signed rank test and Kruskal-Wallis test were performed for nonnormally distributed data. Data were presented as mean \pm standard error of mean (SEM). The statistical analysis involved in this study used a two-sided test, and $p<0.05$ was considered statistically significant.

\section{Results}

IL-22 expression increased in OVA-induced mouse model

$\mathrm{BALB} / \mathrm{c}$ mice were sensitized and challenged by OVA, and treated per-nasally with rmIL-22 or PBS control as described in the methods (Fig. 1a). The ELISA Kit analysis of lung homogenates and BALF demonstrated that OVA-induced asthma, compared with phosphate buffered saline diluent control challenge of the $\mathrm{BALB} / \mathrm{c}$ mice, was associated with significant elevation of the mean concentrations of IL-22 (Fig. 1). The mean concentration of IL-22 was significantly elevated in the OVA-induced mice compared with the control mice treated with PBS challenge in lung homogenates (Fig. 1b, $1.12 \pm 0.06 \mathrm{pg} / \mathrm{mg}$ vs. $0.77 \pm 0.04 \mathrm{pg} / \mathrm{mg}$, $p<0.001)$. Again, this phenomenon similarly occurred in BALF that OVA-induced mice showed a significantly increased mean concentration of IL-22 compared with those challenged with phosphate buffered saline control (Fig. 1c, $9.18 \pm 0.82 \mathrm{pg} / \mathrm{ml}$ vs. $5.23 \pm 0.21 \mathrm{pg} / \mathrm{ml}$, $p<0.01)$.

\section{IL-22 attenuated OVA-induced airways hyperresponsiveness}

To determine the effect of IL-22 on airway function, we exposed $\mathrm{BALB} / \mathrm{c}$ mice to increasing doses of methacholine aerosols. Administration of rmIL-22 generated a significant improvement in the methacholine-induced airways resistance compared with PBS treatment in the OVA-induced asthma model, consistent the previous report [19]. Compared with the PBS-treated control group, the Rrs (Fig. 2a) and Ers (Fig. 2b) values were significantly elevated in OVA-induced mice; meanwhile the Crs values were dramatically decreased (Fig. 2c). However, these phenomena, upregulation of Rrs and Ers and downregulation of Crs, were significantly reversed by 
a

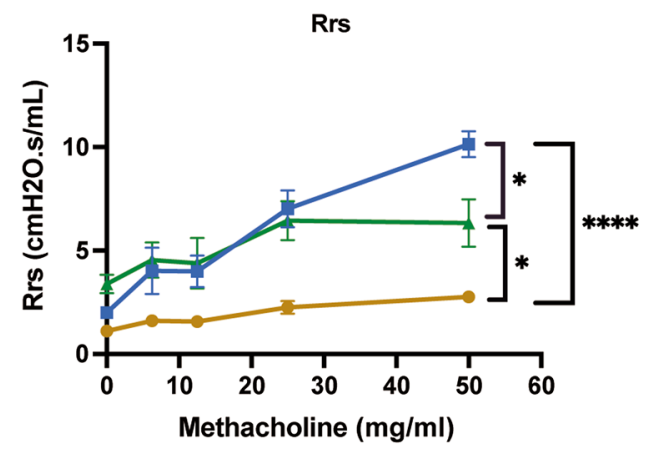

c

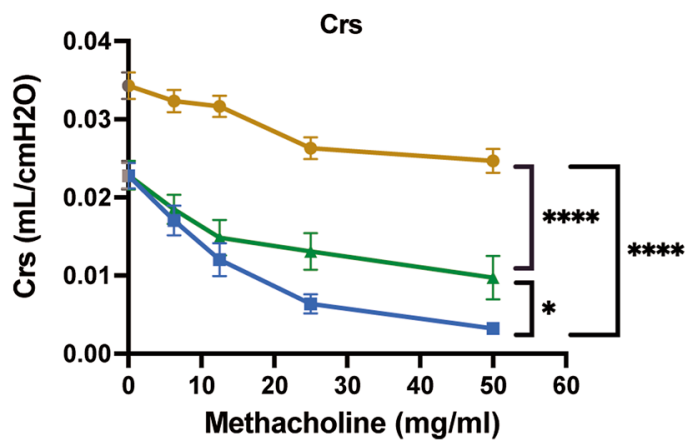

b

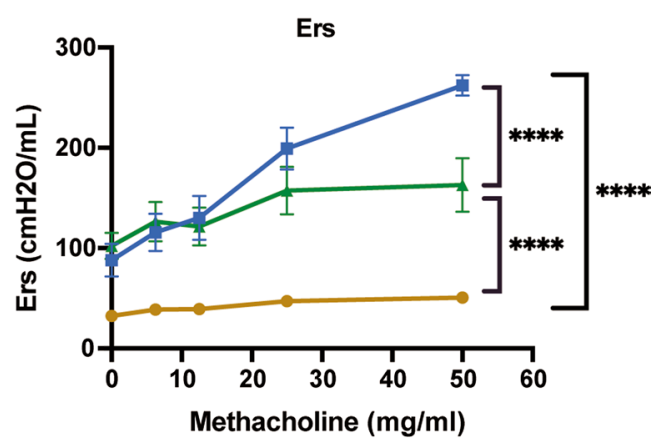

- control

OVA/PBS

$\leftarrow$ OVA/IL-22

Fig. 2 The effects of IL-22 on airway hyperresponsiveness in OVA-induced asthma model. The values of Respiratory system resistance (Rrs) (a), Elastance (Ers) (b), and Compliance (Crs) (c) were measured $24 \mathrm{~h}$ after the last OVA challenge by exposure to increasing doses of methacholine $(\mathrm{n}=8)$. Bars are presented as the mean \pm SEM. ${ }^{*} p<0.05,{ }^{* *} p<0.01,{ }^{* * *} p<0.001$

administration with IL-22, but not completely compared with control group (Fig. 2).

\section{IL-22 attenuated OVA-induced inflammatory cellular infiltration in BALF}

Intranasal challenge with OVA was related with a significant elevation of the mean eosinophils infiltrating the peribronchial and perivascular regions of the lung tissues compared with PBS control, as shown by WrightGiemsa staining, where 400 cells were counted in each section in continuous fields of vision; whereas intranasal administration of rmIL-22 dramatically decreased the mean eosinophils compare with the OVA-induced group (Fig. 3a, $p<0.0001$ ), which was significantly, but not completely attenuated in OVA-induced mice compared with the control mice. We also observed a significant decrease in the mean number of neutrophils and macrophages in BALF of OVA-induced mice, compared with the control group; after IL-22 treatment, the mean number of neutrophils continued to decline compared with the OVAinduced mice (Fig. 3b, $p<0.05$ ), while the mean number of lymphocytes and macrophages were significantly increased in the BALF of the IL-22 treatment group compared with the OVA-induced group (Fig. 3c and d, $p<0.01)$.

\section{IL-22 attenuated OVA-induced eosinophilic infiltration into lung tissue}

Intranasal challenge with OVA induced a significant increase in the total number of cells infiltrating the peribronchial and perivascular areas of the lung tissues, predominantly eosinophils, compared with control group (Fig. 4a). While H\&E staining specimens of the rmIL-22 administration group revealed that the scores of peribronchial and perivascular inflammatory cell infiltration were significantly improved, compared with OVA-induced group (Fig. 4b), but not completely attenuated compared with control group (Fig. 4b).

\section{IL-22 influenced the receptor expression in lung tissues of OVA-induced mouse model}

To explore the potential mechanisms of IL-22 which inhibited the OVA-induced airway inflammation, we performed immunohistochemistry to detect the expression of functional IL-22 receptor, a heterodimer of IL-22RA1 and IL-10RB, and its soluble binding protein, IL-22BP. Immunohistochemistry analysis showed that the 
a
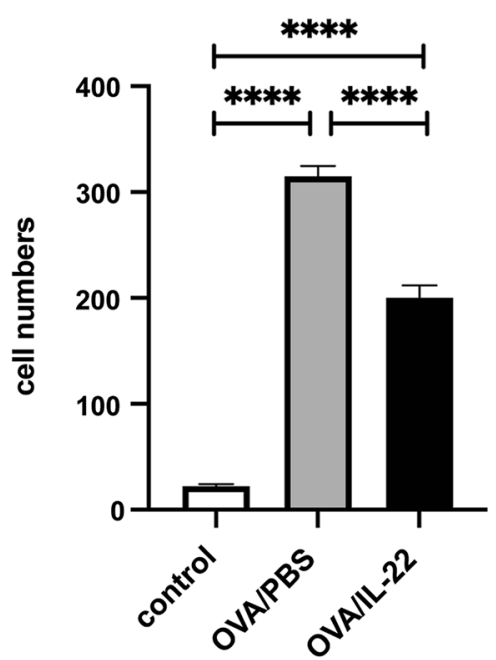

C

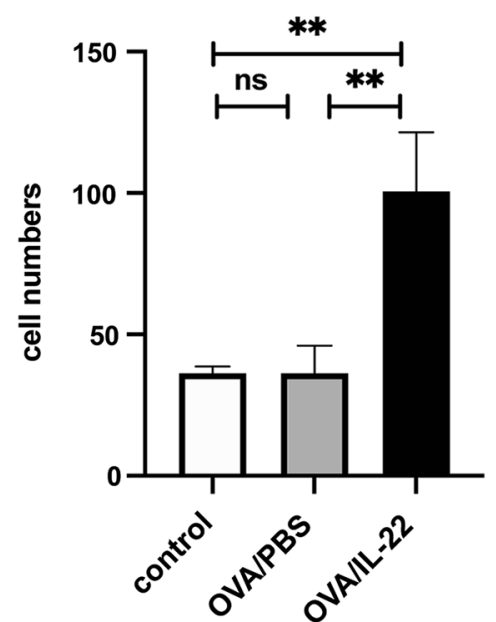

b

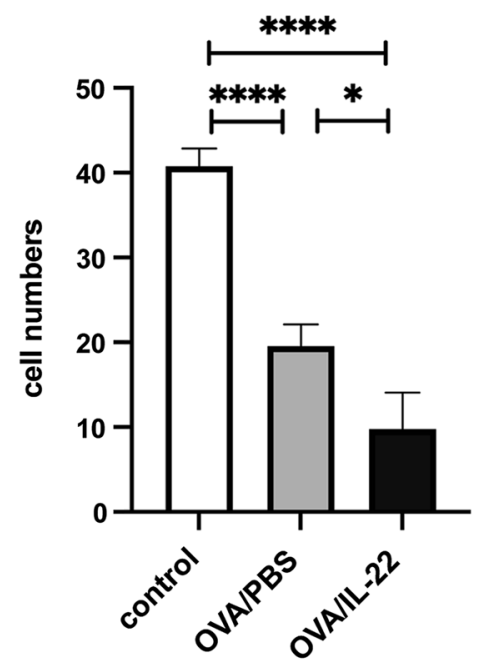

d

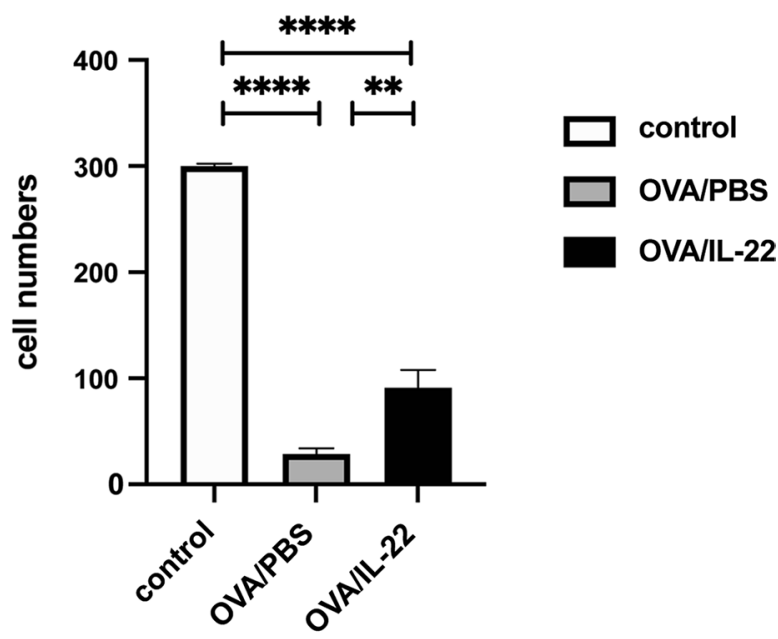

Fig. 3 Intranasal administration of rmlL-22 attenuated OVA-induced eosinophil recruitment into the airways. Recombinant mouse IL-22 (0.2 $\mu \mathrm{g}$ per mouse) or PBS was given intranasally to OVA-induced animals three times in total at $24 \mathrm{~h}$ before the inhaled OVA challenge. Numbers of eosinophils (a), neutrophils (b), lymphocytes $(\mathbf{c})$ and macrophages (d) in BALF at $24 \mathrm{~h}$ after OVA challenge. Bars are presented as the mean \pm SEM $(n=5$ in each group). ${ }^{*} p<0.05,{ }^{* *} p<0.01,{ }^{* *} p<0.001,{ }^{* * *} p<0.0001$

expression of IL-22RA1 and IL-10RB in the lung tissues of OVA-induced mice was significantly increased compared with the control mice (Fig. 5a-c, $p<0.0001$ ), while was dramatically decreased after the treatment with IL-22 with statistical significance (Fig. 5a-c, $p<0.05$ ), but was not completely attenuated in the IL-22-treated mice when compared with the control mice (Fig. 5a-c, $p<0.001)$. We also examined the IL-22BP expression which was significantly enhanced after OVA challenge (Fig. $5 \mathrm{a}$ and d, $p<0.0001$ ), but no difference was noted after IL-22 administration (Fig. $5 \mathrm{~d}, p>0.05$ ).

\section{Discussion}

In this study, we have compared the expression level of IL-22 in OVA-induced mice and control mice, explored the effect of IL-22 on allergic asthma, and found that the IL-22 level significantly increased in lung tissues of the OVA-induced mice and IL-22 showed anti-inflammation properties in allergic asthma, attenuating airway inflammation and airway hyperresponsiveness.

Interleukin-22 is one of the lymphoid cell-derived cytokines, and have exclusive functions on non-hematopoietic cells, especially epithelial cells, playing a critical 


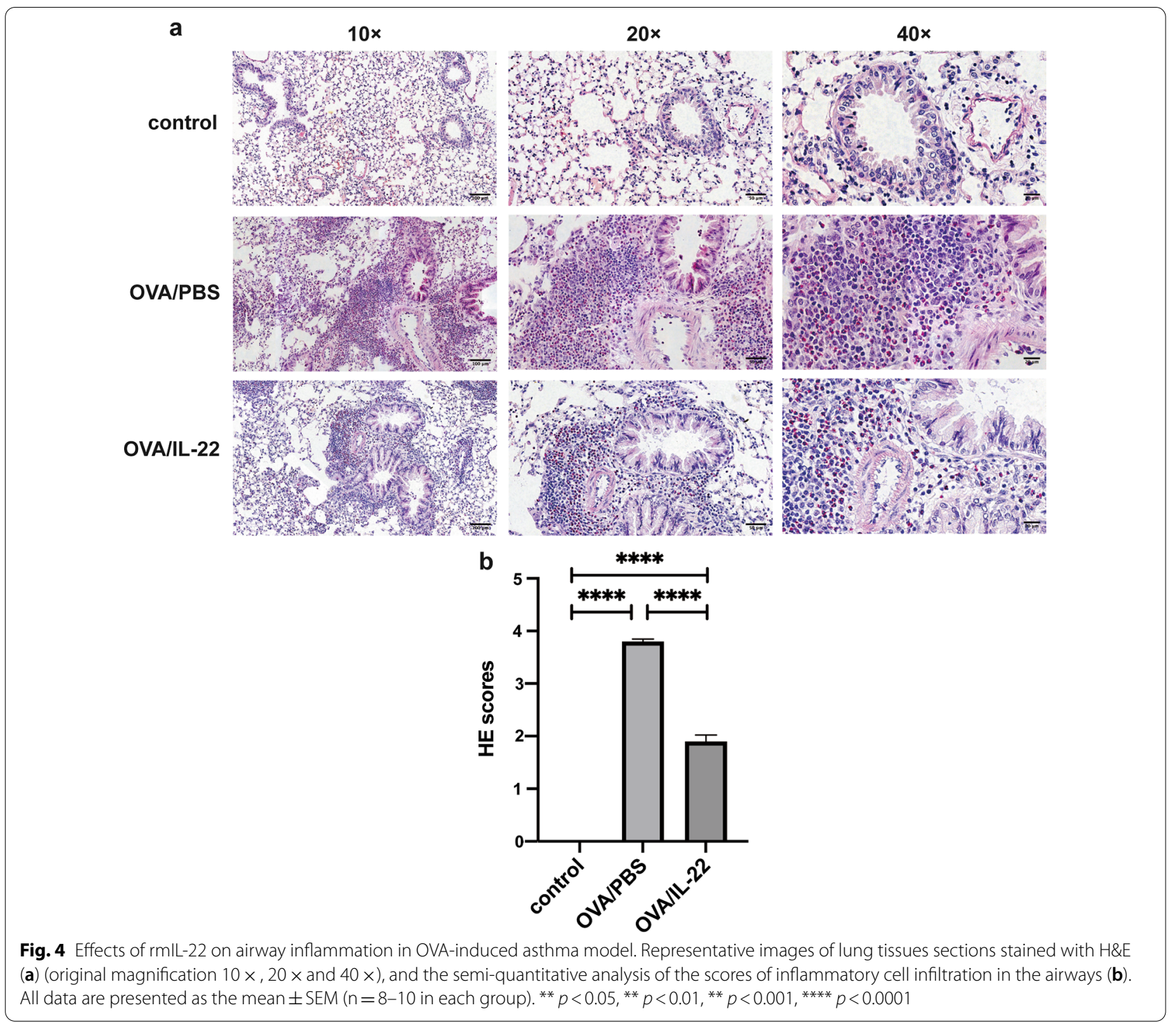

role in the regulation of allergic asthma airway inflammation [11,31]. Previous studies have demonstrated that IL-22 level was higher in allergic asthma than in healthy control $[19,22,32]$. In our study, we detected the IL-22 level in lung homogenates and BALF in allergic asthma model mice and control mice. Consistent with previous findings, the expression level of IL-22 in allergic asthma mice was higher than that in control mice. This observation is firmly supported by that the majority of IL-22 is produced by $\mathrm{CD} 4^{+} \mathrm{T}$ cells in allergic airway inflammation [19]. This may suggest that IL-22 is involved in the pathogenesis of allergic asthma, and further studies are needed to confirm the precise roles of IL-22.

According to previous studies, IL-22 still exhibits controversial properties, both pro-inflammatory and anti-inflammatory, in allergic airway inflammation of murine studies [19, 20, 24, 33]. Previous findings have shown the anti-inflammatory roles of IL-22 in the allergic airway inflammation [19, 20, 34]. Ito et al. have researched that the house dust mite (HDM)-induced allergic airway inflammation and AHR are exacerbated in IL-22 $2^{-/}$mice, indirect exhibiting the protective effect of IL-22 [20]. Fang et al. have generated IL-22 transgenic mice that overexpress IL-22 in the lung, which decreased eosinophils infiltration and reduced AHR in OVA-induced asthma model [34]. Takahashi et al. have adopted with anti-IL-22 antibody, neutralizing IL-22 and enhancing AHR [19]. In our study, we have administrated mice directly with the recombinant murine IL-22 in OVA-induced asthma model mice to investigate the role 


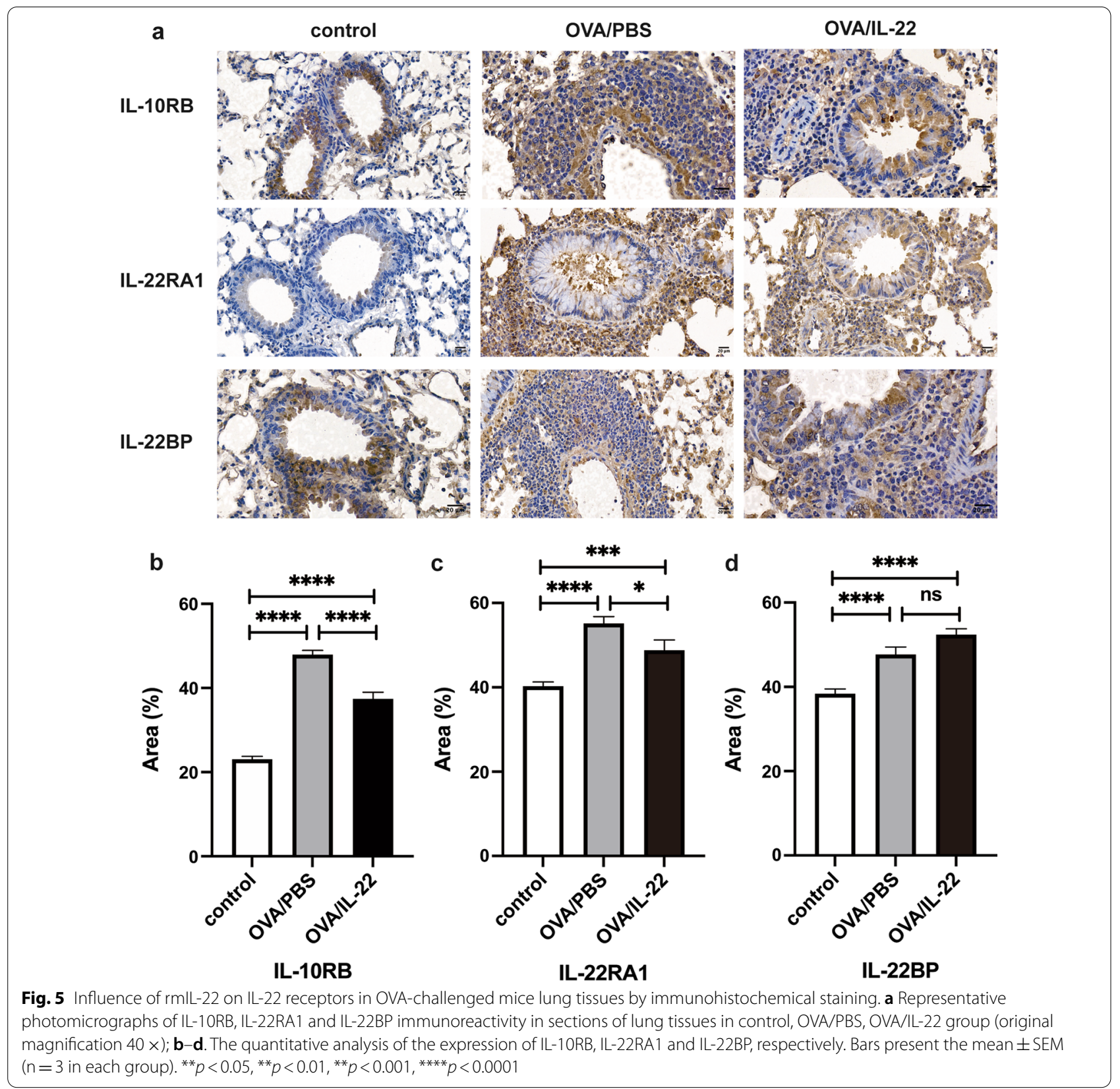

of IL-22, finding that allergic airway inflammation was significantly attenuated by IL-22 administration, including a decreased number of eosinophils in BALF, reduced inflammatory cell infiltration around the bronchi and their accompanying vessels and decreased AHR. Despite of existing some duplication of previous study, this study and previous studies complement each other and jointly illustrate the anti-inflammatory and protective effects of IL-22. These reasons may explain our findings: on the one hand, IL-22 gene delivery inhibited OVA-induced proliferation and cytokine production of $\mathrm{CD}^{+} \mathrm{T}$ cells and increased IL-10 production, an immunosuppressive cytokine [33]; on the other hand, due to predominant acting on lung epithelial cells, IL-22 inhibits the production of epithelial pro-inflammatory cytokines, IL-33, IL-25 and TSLP, by inducing the production of Reg3 $\gamma$, an antimicrobial peptide, in allergic airway inflammation [19, 20]. However, in several previous studies, IL-22 has acted as a pathogenic effect on allergic airway inflammation [22-24, 35]. In skin inflammation and wound healing, IL-22 upregulates the proinflammatory gene expression in a dose-dependent manner, induces the keratinocyte 
migration and inhibits the epidermal differentiation [35]. In the fungi-induced allergic airway inflammation, IL-22 is indispensably required, IL-22 absence attenuates proallergic and proinflammatory responses, and neutralization of IL-22 improves lung function [23]; in addition, in epicutaneous-sensitized and OVA-induced allergic airway inflammation, the generation of $\mathrm{CD} 4^{+} \mathrm{T}$ cells producing IL-22 is promoted, meanwhile, promoting the production of IL-17A and exacerbating airway inflammation and AHR [24]. All above suggested that the protective and pathogenic roles of IL-22 may mainly depend on the routes of sensitization and the antigen species of sensitization and challenge.

Through the heterodimeric receptor consisted by the IL-10RB and the IL-22RA1, IL-22 mediated the downstream STAT3 activation in lung epithelial cells, improving epithelial repair and barrier function, and inducing the production of anti-microbial peptide [20,36, 37]. IL-10RB is widely expressed on hematopoietic and non-hematopoietic cells, while IL-22RA1 is restrictedly expressed on non-hematopoietic cells, especially epithelial cells, determining the target cells of IL-22 [10, 11]. Previous study first reported that IL-22RA1 is expressed on the lung epithelial cells in OVA-induced airway inflammation [19]. Consistent with previous studies, we found that IL-10RB and IL-22RA1 were ubiquitously and restrictedly expressed, respectively; the expression of IL-10RB and IL-22RA1 significantly increased in OVA-induced mice lung tissues, and decreased after the administration of IL-22; but this recovery did not completely reach to the control mice level. Boniface et al. found that blocking IL-10RB and IL-22RA1 could affect the phosphorylation of STAT3, and demonstrated that IL-22 could induce STAT3 phosphorylation through IL10RB and IL-22RA1 [35]. Therefore, it is suggested that IL-22 participates in the allergic airway inflammation by functional IL-22 receptor, IL-10RB and the IL-22RA1 complex. IL-22BP is a soluble binding protein of IL-22 and could inhibit the activity of IL-22, which was significantly increased in OVA-induced mice but no difference was found between the OVA-induced mice group and the IL-22 administration group in our study. IL-22BP gene expression was significantly decreased during influenza infection [38], and there was decreased inflammation, pulmonary injury and pulmonary edema in IL-22BP $\mathrm{BP}^{-1-}$ mice, compared with the wild type mice [39]. These suggest that IL-22BP plays a pro-inflammatory role, and further reveal the protective role of IL-22.

However, there are certain limitations in our study. First, we utilized the wild type mice but not the IL-22 knockout mice to clarify the protective effect on allergic airway inflammation, thus the influence of endogenous IL-22 could not be excluded. Second, we did not detect the effect on production of epithelial inflammatory cytokines. Finally, although we have detected the functional receptors of IL-22, the detailed downstream mechanisms of IL-22 in the OVA-induced asthma model should be further explored in the future studies.

\section{Conclusion}

In summary, the present study clearly demonstrated the protective role of IL-22 in an OVA-induced asthma model. It also showed that IL-22 could suppress the inflammatory cell infiltration around bronchi and their concomitant vessels and airway hyperresponsiveness by its heterodimer receptors. Therefore, IL-22 administration might be an effective strategy to attenuate allergic airway inflammation.

\section{Abbreviations \\ AHR: Airway hyperresponsiveness; ANOVA: One-way analysis of variance; BALF: Bronchoalveolar lavage fluid; $\mathrm{CPH}$ : China Pulmonary Health; Crs: Compliance; ELISA: Enzyme linked immunosorbent assay; Ers: Elastance; HDM: House dust mite; H\&E: Hematoxylin and eosin; IFN: Interferon; IL: Interleukin; ILC3s: Group 3 innate lymphoid cells; IL-22BP: IL-22-binding protein; IL-22RA1: IL-22 receptor A1; Mch: Methacholine; OVA: Ovalbumin; PBMC: Peripheral blood mono- nuclear cells; PBS: Phosphate buffered saline; Rrs: Airways resistance; SEM: Standard error of mean; STAT: Signal transducer and activator of transcription; TSLPL: Thymic stromal lymphopoietin; WT: Wild-type.}

\section{Acknowledgements}

Not applicable.

\section{Authors' contributions}

SG and JZ performed mouse experiments and collected data; $\mathrm{HL}$ and $\mathrm{CL}$ conducted statistical analysis and prepared figures; JW designed the work, performed experiments and wrote the main manuscript text; JL supervised the study and substantively revised the paper. All authors reviewed and approved the final manuscript.

\section{Funding}

This study was funded by The National Key Research and Development Program of China (grant no. 2017-KJBYF-004).

Availability of data and materials

Upon request of corresponding author.

\section{Declarations}

\section{Ethics approval and consent to participate}

All protocols on mice were carried out according to the National Institutes of Health Guidelines for the Care and Use of Laboratory Animals and approved by the Animal Ethical and Welfare Committee of China-Japan Friendship Hospital (approval No. zryhyy21-20-01-3). All methods were conducted in accordance with the ARRIVE guidelines (https://arriveguidelines.org).

\section{Consent for publication}

Not applicable.

Competing interests

All authors declare that they have no conflict of interest.

\section{Author details}

${ }^{1}$ Peking University China-Japan Friendship School of Clinical Medicine, No. 2, East Yinghua Road, Chaoyang Disteict, Beijing 100029, China. ${ }^{2}$ Department of Pulmonary and Critical Care Medicine, China-Japan Friendship Hospital, Beijing 100029, China. ${ }^{3}$ Department of Pulmonary and Critical Care Medicine, 
Graduate School of Chinese Academy of Medical Sciences, Peking Union Medical College, China-Japan Friendship Hospital, Beijing 100730, China.

Received: 25 July 2021 Accepted: 11 October 2021

Published online: 26 November 2021

\section{References}

1. GBD 2015 Chronic Respiratory Disease Collaborators. Global, regional, and national deaths, prevalence, disability-adjusted life years, and years lived with disability for chronic obstructive pulmonary disease and asthma, 1990-2015: a systematic analysis for the Global Burden of Disease Study 2015. Lancet Respir Med 2017;5(9):691-706.

2. Huang K, Yang T, Xu J, Yang L, Zhao J, Zhang X, Bai C, Kang J, Ran P, Shen H, et al. Prevalence, risk factors, and management of asthma in China: a national cross-sectional study. Lancet. 2019:394(10196):407-18.

3. Schatz M, Rosenwasser L. The allergic asthma phenotype. J Allergy Clin Immunol Pract. 2014;2(6):645-8.

4. Soong W, Yoo B, Pazwash H, Holweg CTJ, Casale TB. Omalizumab response in patients with asthma by number and type of allergen. Ann Allergy Asthma Immunol. 2021;127:223-31.

5. Croasdell Lucchini A, Gachanja NN, Rossi AG, Dorward DA, Lucas CD. Epithelial cells and inflammation in pulmonary wound repair. Cells. 2021;10(2):339.

6. Wolk K, Kunz S, Asadullah K, Sabat R. Cutting edge: immune cells as sources and targets of the IL-10 family members? J Immunol. 2002;168(11):5397-402.

7. Ouyang W, O'Garra A. IL-10 family cytokines IL-10 and IL-22: from basic science to clinical translation. Immunity. 2019;50(4):871-91.

8. Spits H, Artis D, Colonna M, Diefenbach A, Di Santo JP, Eberl G, Koyasu S, Locksley RM, McKenzie AN, Mebius RE, et al. Innate lymphoid cells-a proposal for uniform nomenclature. Nat Rev Immunol. 2013;13(2):145-9.

9. Hirose $\mathrm{K}$, Ito T, Nakajima H. Roles of IL-22 in allergic airway inflammation in mice and humans. Int Immunol. 2018;30(9):413-8.

10. Wolk K, Kunz S, Witte E, Friedrich M, Asadullah K, Sabat R. IL-22 increases the innate immunity of tissues. Immunity. 2004;21(2):241-54.

11. Ito T, Hirose K, Nakajima H. Bidirectional roles of IL-22 in the pathogenesis of allergic airway inflammation. Allergol Int. 2019;68(1):4-8.

12. Sonnenberg GF, Fouser LA, Artis D. Border patrol: regulation of immunity, inflammation and tissue homeostasis at barrier surfaces by IL-22. Nat Immunol. 2011;12(5):383-90.

13. Dumoutier L, Lejeune D, Colau D, Renauld JC. Cloning and characterization of IL-22 binding protein, a natural antagonist of IL-10-related T cell-derived inducible factor/IL-22. J Immunol. 2001;166(12):7090-5.

14. Kotenko SV, Izotova LS, Mirochnitchenko OV, Esterova E, Dickensheets H, Donnelly RP, Pestka S. Identification, cloning, and characterization of a novel soluble receptor that binds IL-22 and neutralizes its activity. J Immunol. 2001;166(12):7096-103.

15. Farfariello V, Amantini C, Nabissi M, Morelli MB, Aperio C, Caprodossi S, Carlucc A, Bianchi AM, Santoni G. IL-22 mRNA in peripheral blood mononuclear cells from allergic rhinitic and asthmatic pediatric patients. Pediatr Allergy Immunol. 2011;22(4):419-23.

16. Zhao Y, Yang J, Gao YD, Guo W. Th17 immunity in patients with allergic asthma. Int Arch Allergy Immunol. 2010;151(4):297-307.

17. Tamasauskiene L, Gintauskiene VM, Bastyte D, Sitkauskiene B. Role of IL-22 in persistent allergic airway diseases caused by house dust mite: a pilot study. BMC Pulm Med. 2021;21(1):36.

18. Bullone M, Carriero V, Bertolini F, Folino A, Mannelli A, Di Stefano A, Gnemmi I, Torchio R, Ricciardolo FLM. Elevated serum IgE, oral corticosteroid dependence and IL-17/22 expression in highly neutrophilic asthma. Eur Respir J. 2019;54(5):1900068.

19. Takahashi K, Hirose K, Kawashima S, Niwa Y, Wakashin H, Iwata A, Tokoyoda K, Renauld JC, Iwamoto I, Nakayama T, et al. IL-22 attenuates IL-25 production by lung epithelial cells and inhibits antigen-induced eosinophilic airway inflammation. J Allergy Clin Immunol. 2011;128(5):1067-76.

20. Ito T, Hirose K, Saku A, Kono K, Takatori H, Tamachi T, Goto Y, Renauld JC, Kiyono H, Nakajima H. IL-22 induces Reg3y and inhibits allergic inflammation in house dust mite-induced asthma models. J Exp Med. 2017;214(10):3037-50.

21. Pennino D, Bhavsar PK, Effner R, Avitabile S, Venn P, Quaranta M, Marzaioli V, Cifuentes L, Durham SR, Cavani A, et al. IL-22 suppresses IFN-gammamediated lung inflammation in asthmatic patients. J Allergy Clin Immunol. 2013;131(2):562-70.
22. Besnard AG, Sabat R, Dumoutier L, Renauld JC, Willart M, Lambrecht B, Teixeira MM, Charron S, Fick L, Erard F, et al. Dual Role of IL-22 in allergic airway inflammation and its cross-talk with IL-17A. Am J Respir Crit Care Med. 2011;183(9):1153-63.

23. Lilly LM, Gessner MA, Dunaway CW, Metz AE, Schwiebert L, Weaver CT, Brown GD, Steele C. The $\beta$-glucan receptor dectin-1 promotes lung immunopathology during fungal allergy via IL-22. J Immunol. 2012;189(7):3653-60.

24. Leyva-Castillo JM, Yoon J, Geha RS. IL-22 promotes allergic airway inflammation in epicutaneously sensitized mice. J Allergy Clin Immunol. 2019;143(2):619-30.

25. Hou C, Kong J, Liang Y, Huang H, Wen H, Zheng X, Wu L, Chen Y. HMGB1 contributes to allergen-induced airway remodeling in a murine model of chronic asthma by modulating airway inflammation and activating lung fibroblasts. Cell Mol Immunol. 2015;12(4):409-23.

26. Huang X, Qu D, Liang Y, Huang Q, Li M, Hou C. Elevated S100A4 in asthmatics and an allergen-induced mouse asthma model. J Cell Biochem. 2019;120(6):9667-76.

27. Hoegl S, Bachmann M, Scheiermann P, Goren I, Hofstetter C, Pfeilschifter J, Zwissler B, Muhl H. Protective properties of inhaled IL-22 in a model of ventilator-induced lung injury. Am J Respir Cell Mol Biol. 2011;44(3):369-76.

28. LiY, Wang W, Huang P, Zhang Q, Yao X, Wang J, Lv Z, An Y, Corrigan CJ, Huang $K$, et al. Distinct sustained structural and functional effects of interleukin-33 and interleukin-25 on the airways in a murine asthma surrogate. Immunology. 2015:145(4):508-18.

29. Abdelaziz RR, Elmahdy MK, Suddek GM. Flavocoxid attenuates airway inflammation in ovalbumin-induced mouse asthma model. Chem Biol Interact. 2018:292:15-23.

30. Bandukwala HS, Clay BS, Tong J, Mody PD, Cannon JL, Shilling RA, Verbeek JS, Weinstock JV, Solway J, Sperling Al. Signaling through Fc gamma RIII is required for optimal Thelper type (Th)2 responses and Th2-mediated airway inflammation. J Exp Med. 2007;204(8):1875-89.

31. Dudakov JA, Hanash AM, van den Brink MR. Interleukin-22: immunobiology and pathology. Annu Rev Immunol. 2015;33:747-85.

32. Johnson JR, Nishioka M, Chakir J, Risse PA, Almaghlouth I, Bazarbashi AN, Plante S, Martin JG, Eidelman D, Hamid Q. IL-22 contributes to TGF- $\beta 1$ mediated epithelial-mesenchymal transition in asthmatic bronchial epithelial cells. Respir Res. 2013;14(1):118.

33. Nakagome K, Imamura M, Kawahata K, Harada H, Okunishi K, Matsumoto T, Sasaki O, Tanaka R, Kano MR, Chang H, et al. High expression of IL-22 suppresses antigen-induced immune responses and eosinophilic airway inflammation via an IL-10-associated mechanism. J Immunol. 2011;187(10):5077-89.

34. Fang $P$, Zhou L, Zhou Y, Kolls JK, Zheng T, Zhu Z. Immune modulatory effects of IL-22 on allergen-induced pulmonary inflammation. PLOS ONE. 2014;9(9):e107454

35. Boniface K, Bernard FX, Garcia M, Gurney AL, Lecron JC, Morel F. IL-22 inhibits epidermal differentiation and induces proinflammatory gene expression and migration of human keratinocytes. J Immunol. 2005;174(6):3695-702.

36. Kotenko SV, Izotova LS, Mirochnitchenko OV, Esterova E, Dickensheets H, Donnelly RP, Pestka S. Identification of the functional interleukin-22 (IL-22) receptor complex: the IL-10R2 chain (IL-10Rbeta) is a common chain of both the IL-10 and IL-22 (IL-10-related T cell-derived inducible factor, IL-TIF) receptor complexes. J Biol Chem. 2001;276(4):2725-32.

37. Aujla SJ, Chan YR, Zheng M, Fei M, Askew DJ, Pociask DA, Reinhart TA, MCAIlister F, Edeal J, Gaus K, et al. IL-22 mediates mucosal host defense against gram-negative bacterial pneumonia. Nat Med. 2008;14(3):275-81.

38. Abood RN, McHugh KJ, Rich HE, Ortiz MA, Tobin JM, Ramanan K, Robinson KM, Bomberger JM, Kolls JK, Manni ML, et al. IL-22-binding protein exacerbates influenza, bacterial super-infection. Mucosal Immunol. 2019;12(5):1231-43.

39. Hebert KD, McLaughlin N, Galeas-Pena M, Zhang Z, Eddens T, Govero A, Pilewski JM, Kolls JK, Pociask DA. Targeting the IL-22/IL-22BP axis enhances tight junctions and reduces inflammation during influenza infection. Mucosal Immunol. 2020;13(1):64-74.

\section{Publisher's Note}

Springer Nature remains neutral with regard to jurisdictional claims in published maps and institutional affiliations. 